ARTICLE

\title{
A multivariable Mendelian randomization analysis investigating smoking and alcohol consumption in oral and oropharyngeal cancer
}

\author{
Mark Gormley (1) 1,2,3雨, Tom Dudding (1) 1,2,3, Eleanor Sanderson (iD) 1,3, Richard M. Martin (1) 1,3,4, \\ Steven Thomas ${ }^{2,4}$, Jessica Tyrrell (10 5, Andrew R. Ness ${ }^{4}$, Paul Brennan (1) 6, Marcus Munafò (1) 1,3,7, \\ Miranda Pring ${ }^{2}$, Stefania Boccia ${ }^{8,9}$, Andrew F. Olshan ${ }^{10}$, Brenda Diergaarde ${ }^{11}$, Rayjean J. Hung (1) 12,13, \\ Geoffrey Liu ${ }^{13,14}$, George Davey Smith (1) ${ }^{1,3}$ \& Rebecca C. Richmond (D) 1,3
}

The independent effects of smoking and alcohol in head and neck cancer are not clear, given the strong association between these risk factors. Their apparent synergistic effect reported in previous observational studies may also underestimate independent effects. Here we report multivariable Mendelian randomization performed in a two-sample approach using summary data on 6,034 oral/oropharyngeal cases and 6,585 controls from a recent genomewide association study. Our results demonstrate strong evidence for an independent causal effect of smoking on oral/oropharyngeal cancer (IVW OR $2.6,95 \% \mathrm{Cl}=1.7,3.9$ per standard deviation increase in lifetime smoking behaviour) and an independent causal effect of alcohol consumption when controlling for smoking (IVW OR 2.1,95\% Cl=1.1, 3.8 per standard deviation increase in drinks consumed per week). This suggests the possibility that the causal effect of alcohol may have been underestimated. However, the extent to which alcohol is modified by smoking requires further investigation.

\footnotetext{
${ }^{1}$ MRC Integrative Epidemiology Unit, Population Health Sciences, Bristol Medical School, University of Bristol, Bristol BS8 1TL, UK. ${ }^{2}$ Bristol Dental Hospital and School, University of Bristol, Bristol BS1 2LY, UK. ${ }^{3}$ Department of Population Health Sciences, Bristol Medical School, University of Bristol, Bristol BS8 1TL, UK. ${ }^{4}$ University Hospitals Bristol and Weston NHS Foundation Trust National Institute for Health Research Bristol Biomedical Research Centre, University of Bristol, Bristol BS1 3NU, UK. ${ }^{5}$ RD\&E Hospital, University of Exeter Medical School, RILD Building, Exeter, UK. ${ }^{6}$ Genetic Epidemiology Group, World Health Organization, International Agency for Research on Cancer, Lyon, France. ${ }^{7}$ School of Psychological Science, Faculty of Life Sciences, University of Bristol, Bristol BS8 1TL, UK. ${ }^{8}$ Section of Hygiene, University Department of Life Sciences and Public Health, Università Cattolica del Sacro Cuore, Roma, Italia.

${ }^{9}$ Department of Woman and Child Health and Public Health-Public Health Area, Fondazione Policlinico Universitario A. Gemelli IRCCS, Roma, Italy.

${ }^{10}$ Department of Epidemiology, Gillings School of Global Public Health, University of North Carolina, Chapel Hill, NC 27599, USA. ${ }^{11}$ Department of Human Genetics, Graduate School of Public Health, University of Pittsburgh, and UPMC Hillman Cancer Center, Pittsburgh, PA 15260, USA. ${ }^{12}$ Prosserman Centre for Population Health Research, Lunenfeld-Tanenbaum Research Institute, Sinai Health System, Toronto, Canada. ${ }^{13}$ Dalla Lana School of Public Health, University of Toronto, Toronto, Canada. ${ }^{14}$ Princess Margaret Cancer Centre, Toronto, Canada. ${ }_{\text {email: mark.gormley@bristol.ac.uk }}$
} 
H ead and neck squamous cell carcinoma (HNSCC), which includes cancers of the oral cavity and oropharynx, is the world's 6th most common cancer ${ }^{1}$. Prognosis remains poor, with survival ranging between 19 and $59 \%$ at 10 years $^{2}$. Established risk factors include cigarette smoking and alcohol intake, as well as the human papilloma virus (HPV), which is mainly linked with oropharyngeal cases and thought to be sexually transmitted $^{3-5}$. While a large proportion of cases of head and neck cancer are attributable to the combination of smoking and alcohol, the respective contribution of these risk factors is not clear given the strong association between these behaviours. Better estimation of the known risk effects may help identify or clarify the importance of other potential risk factors. One large pooled analysis ${ }^{6}$ from 17 European and American case-control studies (11,221 cases and 16,168 controls) found that, of the population attributable risk of smoking and alcohol, $4 \%$ could be attributed to alcohol alone and $33 \%$ to tobacco alone, with $35 \%$ explained by a greater than multiplicative joint effect of alcohol and tobacco combined for oral and oropharyngeal cancer. However, the apparent synergistic effect seen in observational studies may underestimate the independent effects of smoking and alcohol.

Mendelian randomisation (MR) is an approach which attempts to minimise issues of measurement error, reverse causation and confounding by using genetic variants which are randomly distributed at birth and are known to be reliably associated with modifiable risk factors of interest, to obtain causal effect estimates for these risk factors on disease outcomes ${ }^{7,8}$. To thoroughly evaluate the causal effects of both alcohol consumption and smoking on the risk of oral and oropharyngeal cancer, we first conducted univariable $\mathrm{MR}$ analysis. A recent genome-wide association study (GWAS) reported a genetic correlation (rg $\sim 0.34,95 \% \mathrm{CI}=0.3,0.4, p=6.7 \times 10^{-63}$ ) between alcohol use and smoking initiation, suggesting that sequence variations overlap substantially and that there is a causal pathway operating between them ${ }^{9}$. To account for this correlation between smoking and alcohol, and to simultaneously investigate the independent effects of each, we conducted multivariable Mendelian randomisation ${ }^{10,11}$. As well as investigating the effects of smoking initiation, we aimed to capture a quantitative lifetime measure of smoking behaviour using the comprehensive smoking index (CSI). This was used to compare the independent causal effect of smoking with the continuous measure of alcoholic drinks per week $^{12}$. Finally, given evidence of strong genetic correlation for both smoking and alcohol intake with other risky behaviours ${ }^{13}$, and the established link between sexual behaviour and HPVdriven head and neck cancer ${ }^{14}$, we conducted additional MR analysis of both risk tolerance and lifetime number of sexual partners on oral cavity (OC) and oropharyngeal cancer (OPC).

In this work we use multivariable MR to demonstrate strong evidence for an independent causal effect of both smoking and alcohol consumption on oral and oropharyngeal cancer. This suggests the possibility that the effect of alcohol may have been previously underestimated. However, the extent to which alcohol is modified by smoking requires further investigation.

\section{Results}

Univariable Mendelian randomisation. Using 176 single nucleotide polymorphisms (SNPs) robustly and independently associated with smoking initiation (Supplementary Data 1), univariable MR provided strong evidence that smoking increases risk of oral/ oropharyngeal cancer (IVW Odds Ratio (OR) 2.5, 95\% $\mathrm{CI}=1.6,3.9, p=6.94 \times 10^{-5}$ per log odds of smoking) (Table 1 , Supplementary Figs. 1, 2). The direction of effect was consistent across the four MR methods tested (IVW, weighted median, weighted mode and MR-Egger), although MR-Egger results had wider confidence intervals (CI) and were less reliable due to low $I^{2}$ values of the SNP-exposure associations, indicating possible violation of the no measurement error (NOME) assumption (Supplementary Table 1). There was some evidence of heterogeneity in the SNP effects (Supplementary Table 2) although no SNP effect outliers were detected from MR-PRESSO and MREgger intercepts indicated limited evidence of directional pleiotropy (Supplementary Table 3). Effects were consistent, and ORs slightly larger, when the summary statistics for smoking initiation were obtained from a GWAS meta-analysis in GSCAN which excluded 23andMe and UK Biobank (IVW OR 3.3, 95\% CI = 1.8, 6.1) and a GWAS conducted in the UK Biobank only (IVW OR $5.2,95 \% \mathrm{CI}=1.8,14.9)($ Table 1$)$.

Findings of a causal effect of smoking initiation on oral and oropharyngeal cancer risk were further supported by MR analysis using 108 SNPs associated with a lifetime measure of smoking behaviour, the comprehensive smoking index (CSI) (Supplementary Data 1). Here, a 1 standard deviation (SD) increase in the CSI (equivalent to an individual smoking 20 cigarettes a day for 15 years and stopping 17 years ago, or an individual smoking 60 cigarettes a day for 13 years and stopping 22 years ago) was found to increase oral and oropharyngeal cancer risk combined, with an IVW OR of $3.5\left(95 \% \mathrm{CI}=2.4,5.0, p=5.97 \times 10^{-11}\right)$ (Table 1 , Supplementary Figs. 3, 4, Supplementary Tables 1-3).

Using 60 SNPs robustly and independently associated with number of alcoholic drinks per week, univariable MR provided strong evidence that increased alcohol consumption increases risk of oral/oropharyngeal cancer. Here a 1 SD increase in drinks per week (equivalent to 9 additional drinks per week) was found to increase oral and oropharyngeal cancer risk combined, with an IVW OR of $10.0\left(95 \% \mathrm{CI}=5.3,18.6, p=5.64 \times 10^{-13}\right)($ Table 2$)$. The direction of effect was consistent across the four MR methods tested and high $I^{2}$ values of the SNP-exposure associations indicated low bias from regression dilution (i.e., attenuation towards the null of an association as a result of random measurement error) (Supplementary Table 1). However, there was some heterogeneity in the SNP effect estimates (Supplementary Table 2) and MR-Egger intercepts indicated some evidence for directional pleiotropy (Supplementary Table 3). Effects were consistent, although ORs slightly smaller, when the summary statistics for drinks per week were obtained from a GWAS metaanalysis in GSCAN which excluded 23andMe and UK Biobank (IVW OR 8.3, 95\% CI $=4.7,14.4$ ) and a GWAS conducted in the UK Biobank only (IVW OR 5.8, 95\% CI = 3.7, 9.0) (Table 1). In the main analysis, MR-PRESSO identified an influential outlier which was the $A D H 1 B$ variant, $r 1229984$ (outlier test $p$-value $=$ 0.024 ) which was also shown to be a clear outlier in both scatter and leave-one-out plots (Fig. 1). With removal of this outlier, the causal effect estimate was halved but still large (OR 4.5, 95\% CI = $2.3,9.0)$. The MR-Egger intercept indicated no further directional pleiotropy once this variant was removed (Supplementary Table 3).

Stratification by cancer subsite. In MR analysis stratified by cancer subsite, we found evidence for a causal effect of smoking initiation in both oral cavity (IVW OR 2.0, 95\% CI = 1.2, 3.4) and oropharyngeal cancer (IVW OR 2.8, 95\% CI =1.6, 4.9); comprehensive smoking index in OC (IVW $2.6,95 \% \mathrm{CI}=1.6,4.3$ ) and OPC (IVW 4.0, 95\% CI = 2.5, 6.5); and alcoholic drinks per week in OC (IVW OR 5.9, 95\% CI 2.4, 14.2) and OPC (IVW OR $3.3,95 \% \mathrm{CI}=1.3,8.1$, which were consistent across the $\mathrm{MR}$ methods (Fig. 2).

Multivariable Mendelian randomisation. In the multivariable MR analysis controlling for alcohol consumption, there was 


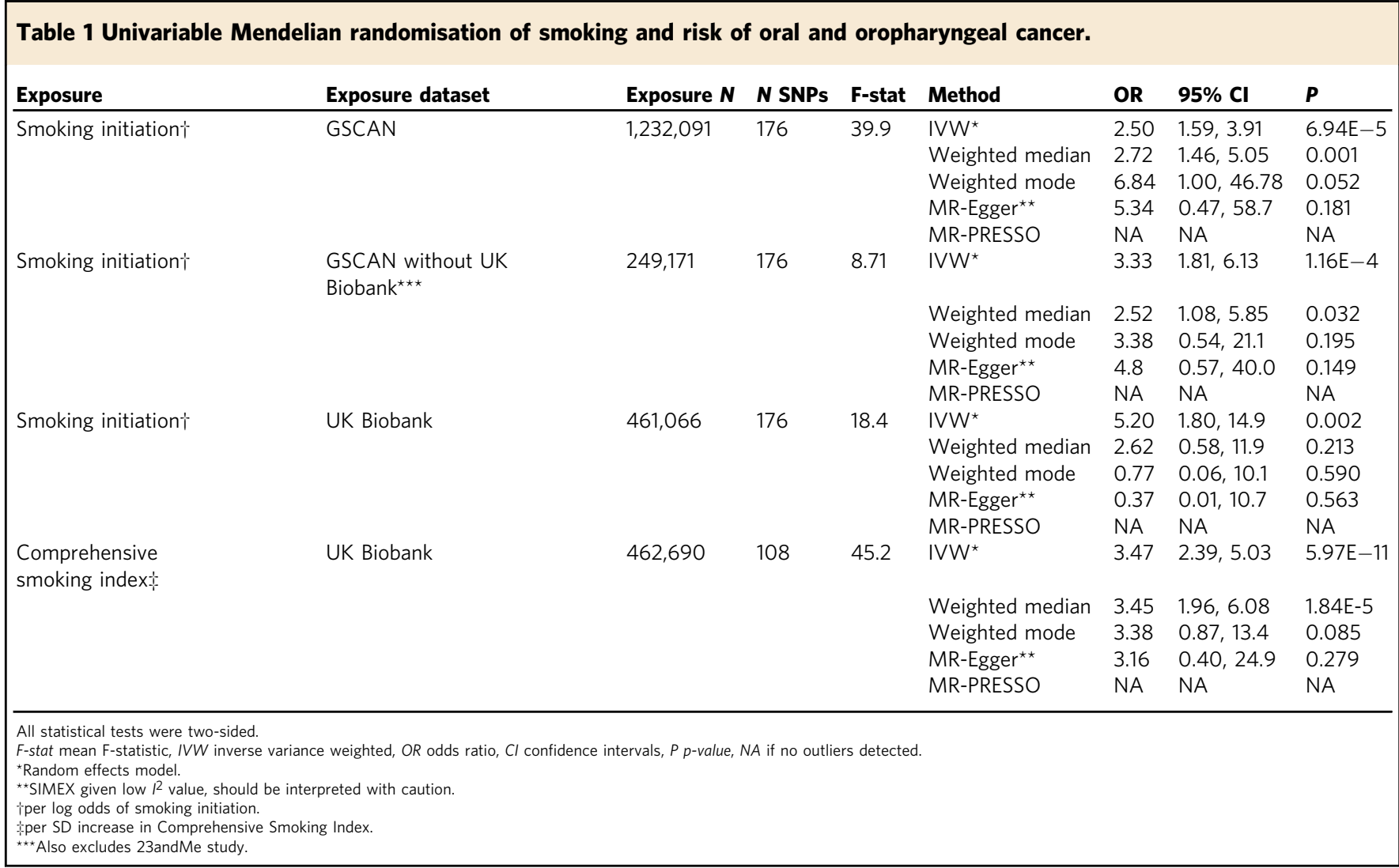

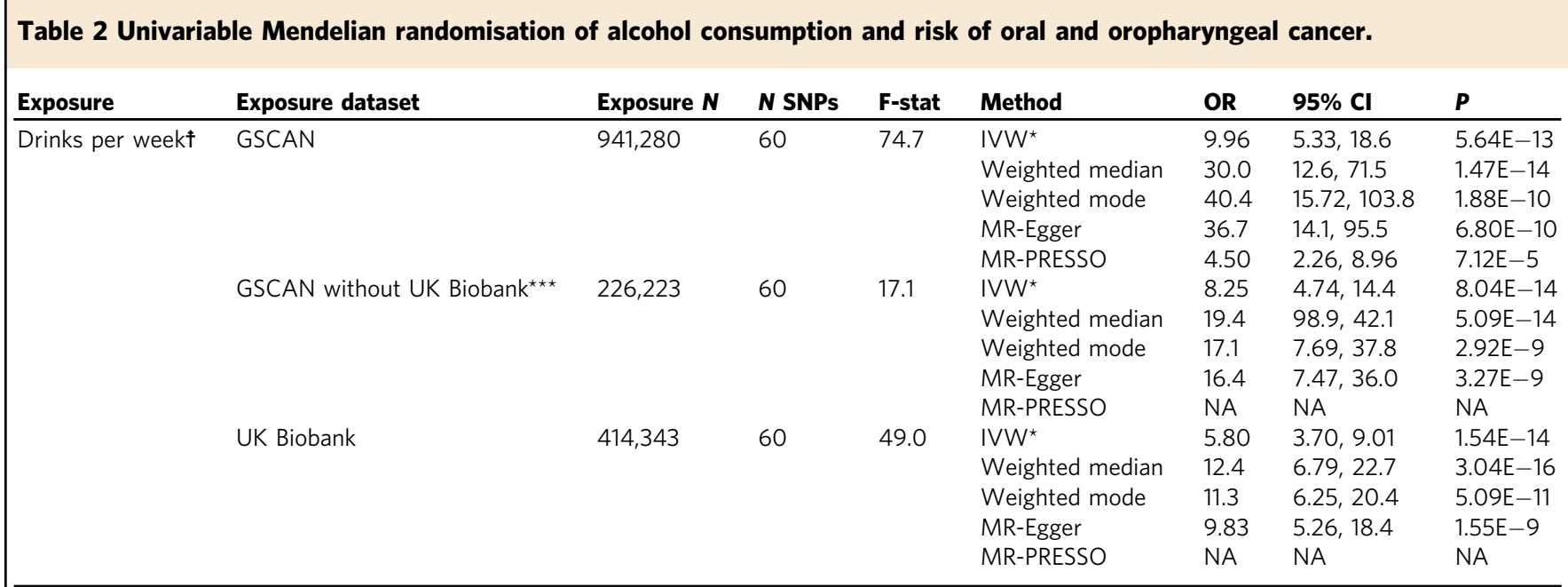

All statistical tests were two-sided.

F-stat mean F-statistic, IVW inverse variance weighted; OR odds ratio, $\mathrm{Cl}$ confidence intervals, $\mathrm{P} p$-value, NA if no outliers detected.

${ }^{\star}$ Random effects model.

†per SD increase in drinks per week

${ }^{\star \star \star}$ Also excludes 23 andMe study.

strong evidence for a direct causal effect of lifetime smoking behaviour on risk of oral/oropharyngeal cancer (IVW OR 2.6, $95 \% \mathrm{CI}=1.7,3.9$ per SD increase in the CSI) (Table 3 ). In multivariable $\mathrm{MR}$ analysis controlling for lifetime smoking, there was also strong evidence for a direct causal effect of alcohol consumption on risk of oral/oropharyngeal cancer (IVW OR 5.2, 95\% CI $=3.2,8.6$ per SD increase in drinks per week) (Table 3). The independent causal effects estimated from multivariable MR-Egger were consistent with the IVW analysis for both alcohol consumption and lifetime smoking (Table 3). While there was limited evidence for heterogeneity in the SNP effect estimates indicating instrument validity, the MR-Egger intercept deviated from the null, which is suggestive of directional pleiotropy. When we removed the $A D H 1 B$ variant found 
a

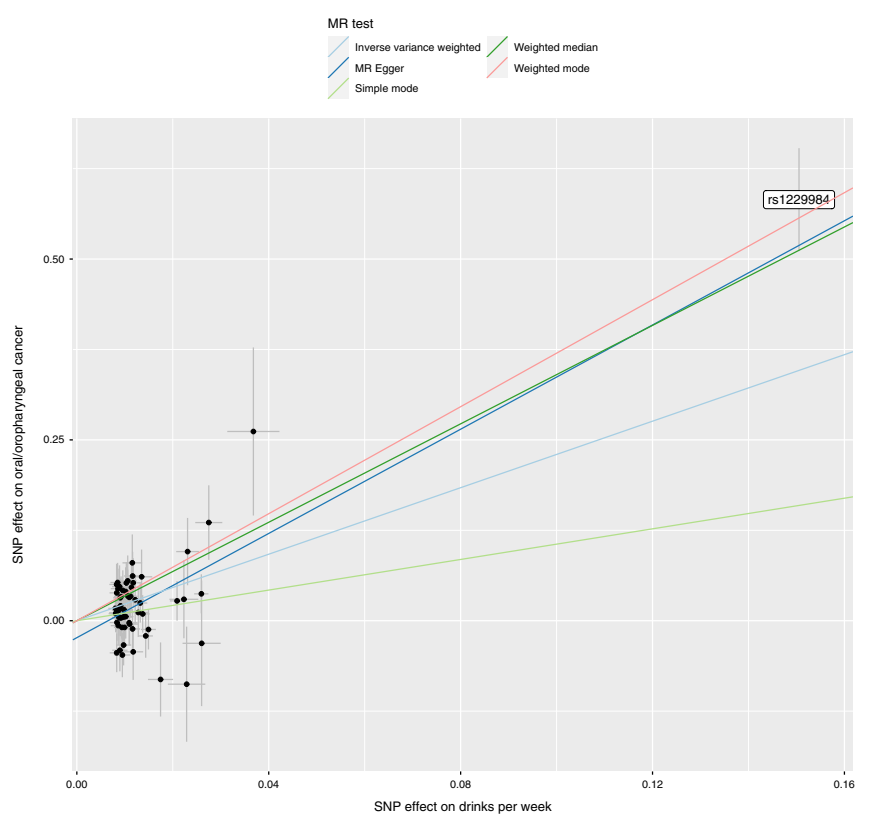

b

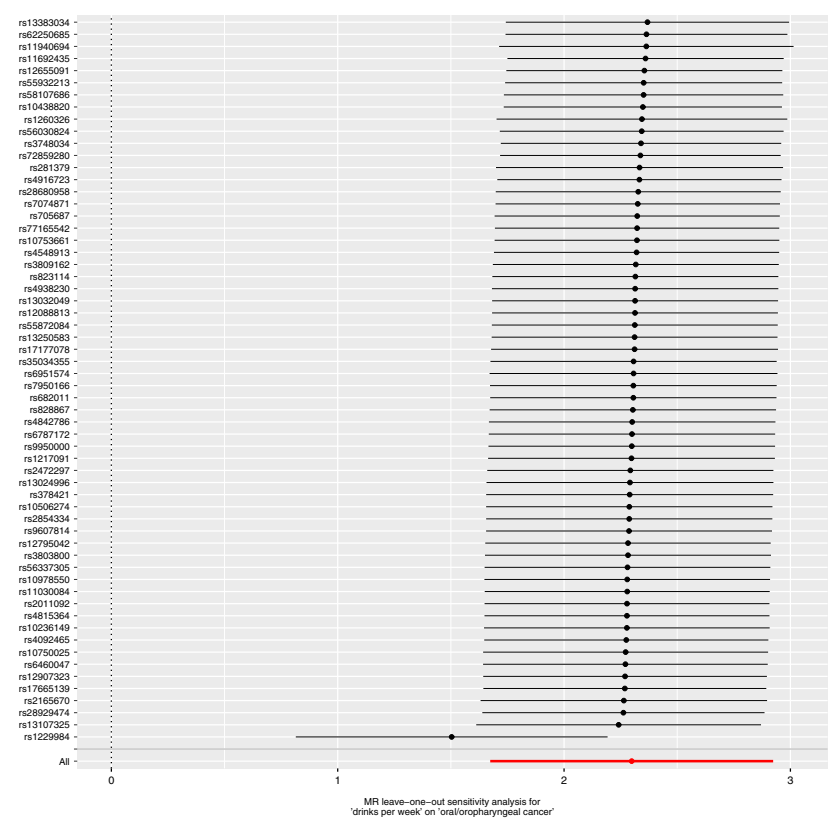

Fig. 1 Scatter and leave one out plots demonstrating influential outliers in univariable MR of alcohol consumption and oral and oropharyngeal cancer risk. a Scatter plot showing $A D H 1 B$ (rs1229984), an outlier single nucleotide polymorphism in the analysis of alcohol consumption (drinks per week, $n=941,280)$ and oral and oropharyngeal cancer risk $(n=6034$ cases and 6585 controls). b Leave one out plot again demonstrating the $A D H 1 B$ (rs1229984) influential outlier. Consistent evidence for a causal effect of alcohol on oral and oropharyngeal cancer risk was found even when this variant was excluded from the analysis. Effect estimates are reported per SD increase in the exposure and error bars represent $95 \%$ confidence intervals.
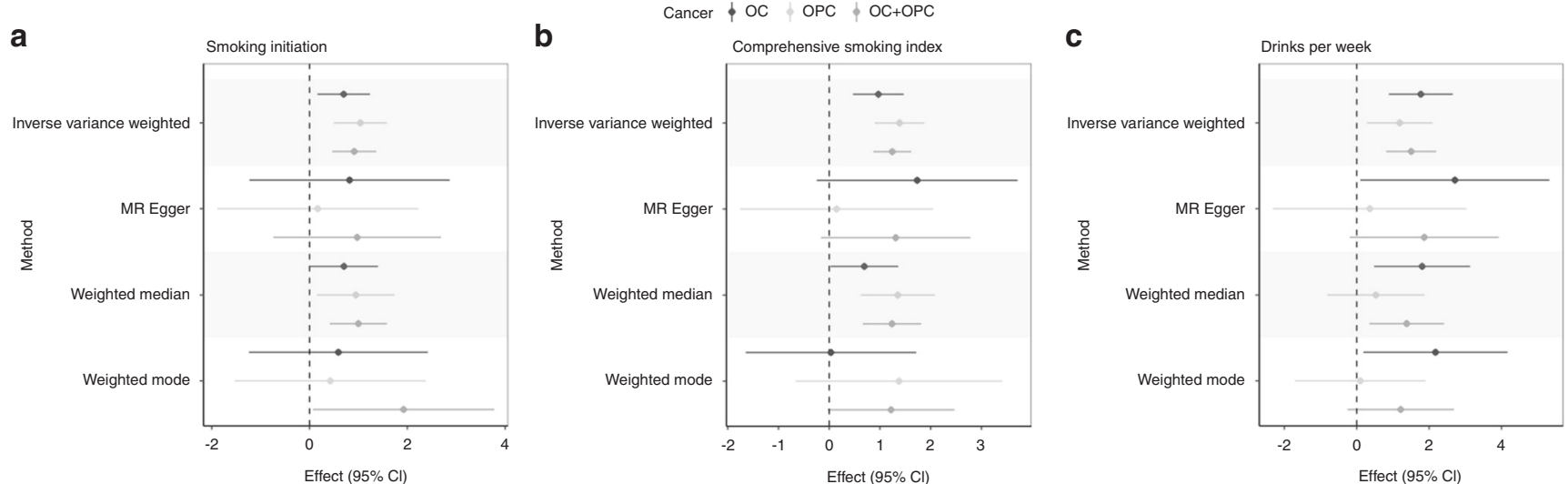

Fig. 2 Univariable Mendelian randomisation of smoking initiation, lifetime smoke exposure and drinks per week on oral and oropharyngeal cancer subsites. Univariable effects displayed were obtained using summary-level data from the GWAS of (a) smoking initiation ( $n=1,232,091)$, (b) comprehensive smoking index $(n=462,690)$ and (c) drinks per week $(n=941,280)$ on oral and oropharyngeal cancer risk $(n=6034$ cases and 6585 controls). Smoking initiation estimates are reported per log odds increase, while comprehensive smoking index and drinks per week are reported per SD increase in drinks per week. Error bars represent 95\% confidence intervals. All statistical tests were two-sided.

to be an outlier in the univariable MR analysis, the independent effects of both smoking and alcohol on risk of oral/oropharyngeal cancer persisted remained, although the magnitude of the effect was reduced for alcohol (IVW OR 2.1, 95\% CI $=1.1$, 3.8) (Table 3, Fig. 3). The MR-Egger intercept indicated no further directional pleiotropy once this variant was removed (Supplementary Table 3).

In multivariable MR analysis stratified by cancer subsite, we found evidence for a causal independent effect of lifetime smoking in OC (IVW OR 2.5, 95\% CI = 1.5, 4.1) and OPC (IVW 3.7, 95\% CI = 2.3, 6.0); and for alcoholic drinks per week in
OC (IVW OR 2.2, 95\% CI $=1.0,5.0)$ and OPC (IVW OR 1.9, $95 \% \mathrm{CI}=0.9,4.0)$ (Fig. 3).

Investigating other risky behaviours. We found limited evidence to suggest there is a causal effect of risk tolerance more generally on risk of oral and oropharyngeal cancer (IVW OR 1.0, 95\% $\mathrm{CI}=0.6,1.7$ per SD) which was consistent across the MR methods applied (Fig. 4). There was some evidence for a causal effect of lifetime number of sexual partners (IVW OR 1.5, 95\% $\mathrm{CI}=1.0,2.3$ per SD), which was specific to oropharyngeal (IVW OR $2.2,95 \% \mathrm{CI}=1.3,3.8$ per $\mathrm{SD}$ ) rather than oral cavity cancer 


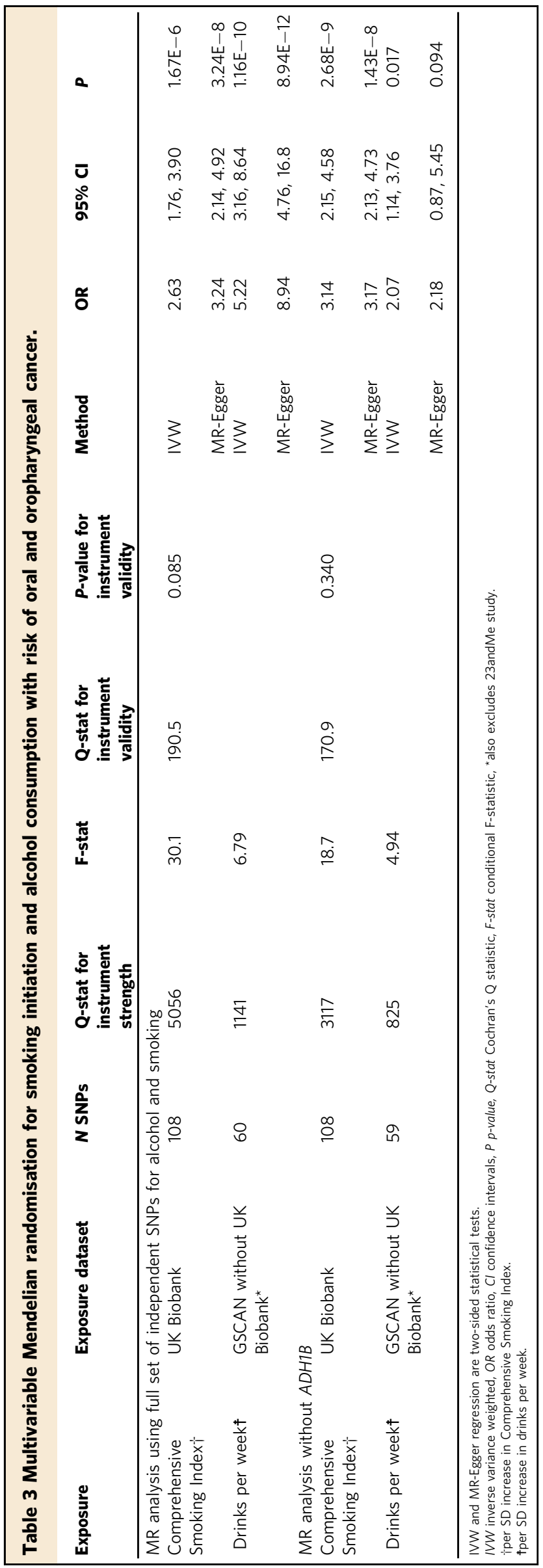

risk (IVW OR 1.2, 95\% CI $=0.7,2.0$ ), and reasonably consistent across the MR methods applied (Fig. 4).

\section{Discussion}

In this study, we applied both univariable and multivariable Mendelian Randomisation to determine a causal, independent effect of both smoking and alcohol consumption on the risk of oral and oropharyngeal cancer. The results were largely robust to sensitivity analyses accounting for horizontal pleiotropy. Effects were consistent between both oral cavity and oropharyngeal subsites and unlikely to be strongly influenced by risk tolerance and lifetime number of sexual partners.

Supporting of our findings regarding an independent effect of smoking, average relative risks (RR) of reported tobacco smoking have been found to range from 4.0-5.0 for both the oral cavity and oropharynx across 16 case-control and 3 cohort studies ${ }^{15}$. Alcohol use has been associated with an increased risk of oral and oropharyngeal cancer in a dose-dependent manner (RR 1.1 (95\% $\mathrm{CI}=1.0,1.3)$ for light drinking to $\mathrm{RR} 5.1(95 \% \mathrm{CI}=4.3,6.1)$ for heavy drinking $(>50 \mathrm{~g} \text { alcohol per day })^{16}$. Hashibe et $\mathrm{al}^{6}$. investigated the independent effects of smoking and alcohol and found an OR of $2.4(95 \% \mathrm{CI}=1.7,3.4)$ for ever tobacco use among never alcohol drinkers and a more than multiplicative joint effect of tobacco and alcohol, but no clear effect of ever alcohol use among never tobacco users ( $\mathrm{OR} 1.1,95 \% \mathrm{CI}=0.9,1.3)$, suggesting that alcohol use on its own may not play an important role ${ }^{6}$. Limitations of observational studies include recall bias, differential measurement error, as well as potential residual confounding, for example by socio-economic position, HPV status $^{17}$ and other lifestyle factors ${ }^{18-20}$.

The independent effect for alcohol in our study suggests the possibility that previous observational estimates for alcohol may have been underestimated, but these cannot be directly compared given the differences in the methodological approaches and interpretation of estimates. MR estimates may reflect the effects of lifelong alcohol exposure, in comparison to the short-term effects captured in observational studies. Furthermore, while multivariable MR can estimate the independent (and unconfounded) effect of alcohol on oral/oropharyngeal cancer risk, it cannot determine the extent to which this effect is modified by smoking status, i.e., is it observed among smokers only or the whole population. Further individual level data analysis is required to determine this.

Several mechanisms have been suggested to explain the observed associations between alcohol and risk of head and neck cancer, including contaminants present in alcoholic drinks (e.g., $\mathrm{N}$ nitrosodiethylamine in beer $)^{21}$, free radical damage and impairment of DNA repair capacity 22,23 . Ethanol is oxidised to acetaldehyde, which has a direct carcinogenic effect and moreover alcohol may act as a 'solvent' for tobacco carcinogens and the induction of carcinogen-metabolising enzymes ${ }^{24,25}$. Given their strong co-existence, the effects of smoking and alcohol are often difficult to separate out. However, in addition to the reported synergistic effect, previous observational data has highlighted an independent effect of both agents ${ }^{26}$, with our results supporting this.

Studies which have used genetic variation in alcoholmetabolising genes do give some support to our findings. For example, East Asians who are homozygous for the $(* 2 * 2)$ variant allele of $A L D H 2$ (aldehyde dehydrogenase 2) are unable to metabolise acetaldehyde, which deters them from drinking alcohol, whereas those who are heterozygous $\left({ }^{*} 1 * 2\right)$ have a 6 -fold higher blood acetaldehyde concentration post-alcohol consumption with respect to the wild type $*_{1} * 1$. The OR of HNSCC among individuals with $* 2 * 2$ has been found to be $0.5(95 \% \mathrm{CI}=$ $0.3,1.0)$ and $1.8(95 \% \mathrm{CI}=1.2,2.8)$ among those with $* 1 * 2$, relative to $*^{*} 1$ individuals. These findings support the theory 


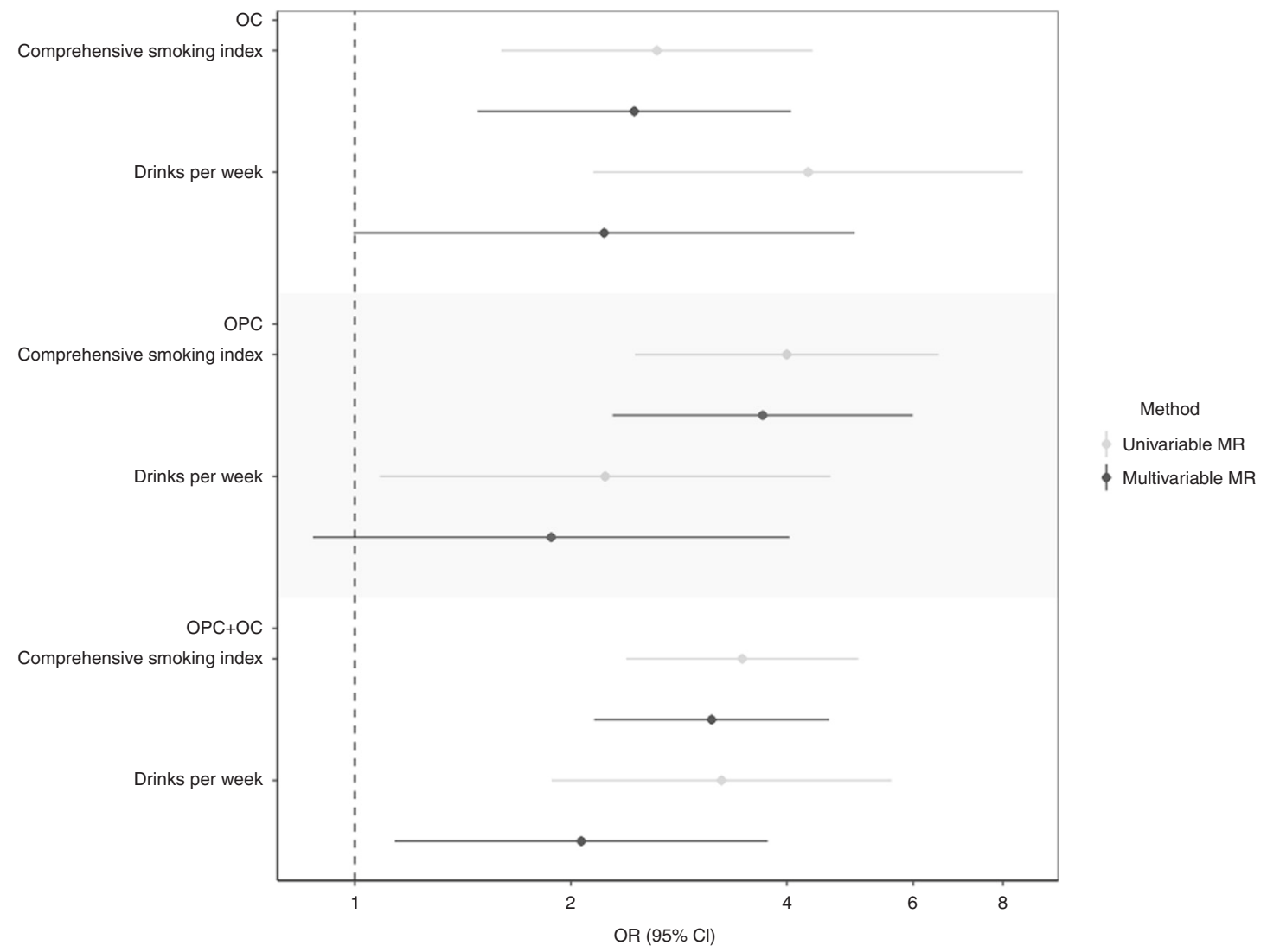

Fig. 3 Multivariable Mendelian randomisation of lifetime smoke exposure and drinks per week on oral and oropharyngeal subsites. Effect

estimates (ORs) are reported per SD increase in the exposure of drinks per week $(n=226,223)$ and the comprehensive smoking index $(n=226,223)$ on oral and oropharyngeal cancer risk $(n=6034$ cases and 6585 controls). Error bars represent $95 \%$ confidence intervals. Genetic instrument for drinks per week excludes outlying variant, $A D H 1 B$ (rs1229984). Univariable and multivariable effects displayed were obtained using summary-level data from the GWAS of comprehensive smoking index in UK Biobank and summary-level data from a GWAS of drinks per week in GSCAN, excluding UK Biobank and 23andMe. All statistical tests were two-sided.

Cancer $\downarrow O C \$ O P C \nmid O C+O P C$

a

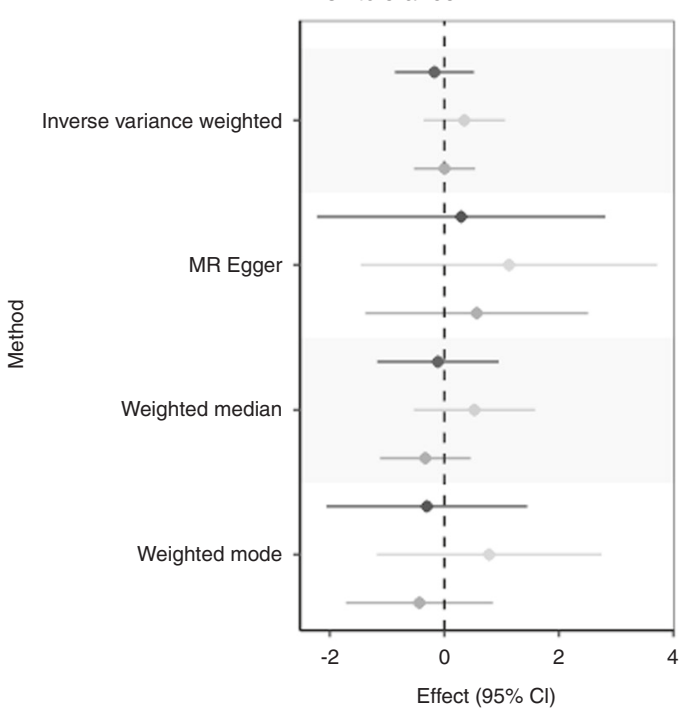

b

Number of sexual partners

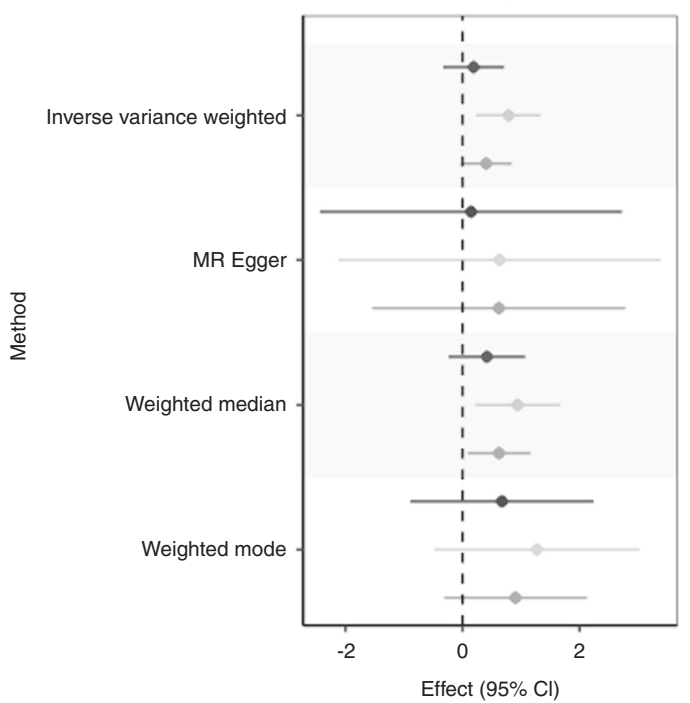

Fig. 4 Univariable Mendelian randomisation of risk tolerance and number of sexual partners on oral and oropharyngeal cancer subsites. Effect estimates (log odds) are reported per SD increase in (a) risk tolerance $(n=508,782)$ and (b) number of sexual partners $(n=370,711)$. There was some evidence for a causal effect of number of sexual partners specific to the oropharyngeal subsite. Error bars represent $95 \%$ confidence intervals. All statistical tests were two-sided. 
that alcohol increases HNSCC risk given the protective effect of non-drinking among homozygous individuals, while also indicating the carcinogenic action of acetaldehyde ${ }^{27}$. While the frequency of the $* 2$ variant of $A L D H 2$ in non-Eastern populations is low, a recent GWAS revealed variation at other sites in the genome which are linked with alcohol consumption ${ }^{9}$. An MR analysis using these multiple variants robustly associated with alcohol from this recent GWAS $^{9}$, demonstrated a causal effect of alcohol intake on HNSCC (OR 3.9, 95\% CI $=1.3,11.2)$ in the UK Biobank, although there were only 856 HNSCC cases included in this cohort $^{28}$. A more recent multivariable MR study in UK Biobank $^{29}$ investigated smoking initiation and alcohol consumption with multiple cancers. This study demonstrated that smoking initiation increased odds of head and neck cancer when adjusted for alcohol (IVW OR 1.4, 95\% CI $=1.1,1.8, p=0.002$ ). There was also a strong positive effect of alcohol consumption on head and neck cancer risk when adjusted for smoking (IVW OR $1.9,95 \% \mathrm{CI}=0.8,4.6, p=0.142$ ), although with wider confidence intervals ${ }^{29}$.

The current study applied both univariable and multivariable MR methods, using the largest number of SNPs identified from the latest GWAS for both smoking9,12, alcohol consumption 9 ,13 and head and neck cancer ${ }^{30}$, that could be identified in the literature. A series of pleiotropy-robust MR methods and outlier detection were applied to rigorously explore the possibility that findings were not biased as a result of pleiotropy. Comparability of smoking initiation and alcohol consumption is an issue given that alcohol consumption is closer to a measure of lifetime use, whereas smoking initiation captures both light and short-term smokers and heavy, long-term smokers. To account for this and to evaluate the causal effects of a quantitative measure of smoke exposure, we performed univariable and multivariable MR using genetic variants identified in relation to a $\mathrm{CSI}^{12}$.

The effect of smoking and alcohol on HNSCC risk is thought to be synergistic, with their combined use interacting in a multiplicative manner ${ }^{31}$. We were not able to investigate this here as MR interaction analysis relies on individual-level data which we did not have access to ${ }^{32}$. We were also unable to investigate the potential carcinogenic role of acetaldehyde, distinct from alcohol intake, as has been done previously using alcohol dehydrogenase genotypes $^{27,33}$, as this again typically relies on individual-level data to assess gene-by-environment interactions. Our finding that the $A D H 1 B$ variant, rs1229984 was a clear outlier which inflated causal estimates, indicates pleiotropy of this variant via its role in alcohol metabolism. However, we found consistent evidence for a causal effect of alcohol on oral and oropharyngeal cancer risk when this variant was excluded from the analysis.

While we used a large number of genetic variants to serve as stronger instruments for both smoking and alcohol, these variants have not all been well characterised and indeed some of the top loci have been previously associated with other risky behaviours ${ }^{13}$. Therefore, additional analyses to evaluate the causal effects of risk tolerance and lifetime number of sexual partners on oral/oropharyngeal cancer were conducted. While there was no strong evidence for a causal effect of risk tolerance more generally, an effect of lifetime number of sexual partners was observed, specific only to oropharyngeal cancer risk. This effect is likely mediated through HPV and thought to be sexually transmitted ${ }^{4,5}$. While further work is required to investigate this effect of HPV status among oropharyngeal sites, it is unlikely to have substantially biased the MR estimates for smoking and alcohol, since no effect of lifetime number of sexual partners was observed in relation to oral cavity cancer risk, whereas similar effect estimates were observed in relation to alcohol and smoking. Finally, as most participants in the GAME-ON network were of European or North American decent, with only 10.8\% from
South America, more work is required to determine if our results translate to other ancestry groups.

In conclusion, this study used both univariable and multivariable MR analyses to demonstrate an independent causal effect for both smoking and alcohol on oral and oropharyngeal cancer risk. In particular, we observed large effects for alcohol in our multivariable MR analysis, which suggests the possibility that previous observational estimates for alcohol may have been underestimated. However, these cannot be directly compared given the differences in the methodological approaches and interpretation of estimates. Further work using individual-level data could provide more robust evidence for a synergistic effect and the carcinogenic role of acetaldehyde. Findings from this study add to a growing body of evidence from MR studies surrounding the harmful effects of alcohol consumption ${ }^{34}$ and should be used to guide public health messages regarding the harms of even moderate drinking.

\section{Methods}

Univariable and multivariable Mendelian randomisation were applied using summary-level genetic data from the GWAS and Sequencing Consortium of Alcohol and Nicotine use (GSCAN) ${ }^{9}$, the UK Biobank study ${ }^{12,13}$, and a GWAS of oral and oropharyngeal cancer conducted by the Genetic Associations and Mechanisms in Oncology (GAME-ON) Network ${ }^{30}$, in a two-sample MR framework $^{35}$. Mendelian randomisation is an approach which uses genetic variants as instruments to obtain estimates for the causal effect of these risk factors on disease outcomes. Three assumptions must be satisfied to ensure an MR study is valid which include: (1) genetic variants should be robustly associated with the risk factor of interest (i.e., the relevance assumption), (2) there are no confounders of the genetic variants-outcome association (the independence assumption) and (3) the exclusion restriction assumption. For further detail on the terms used in this study please read the Mendelian randomisation dictionary ${ }^{36}$.

Summary-level data from GSCAN and UK Biobank. Summary-level genomewide association studies were obtained for alcohol consumption (drinks per week, $n=941,280$ ) and smoking initiation (a binary phenotype indicating whether an individual had ever smoked regularly) $(n=1,232,091)$ from the GSCAN study 9 . The comprehensive smoking index (CSI) was derived by Wootton et al in the UK Biobank $(n=462,690)^{12}$. This included information on smoking duration, heaviness and cessation, which were combined into a lifetime smoking index along with a simulated half-life $(\tau)$ constant. Summary-level GWAS data were obtained for this phenotype in the UK Biobank ${ }^{12}$.

Summary-level data from GAME-ON. GWAS was performed on 6,034 HNSCC cases and 6,585 controls from 12 studies which were part of the GAME-ON network ${ }^{30}$. Cancer cases comprised the following ICD-10 codes: oral cavity (C02.0-C02.9, C03.0-C03.9, C04.0-C04.9 and C05.0-C06.9) and oropharynx (C01.9, C02.4 and C09.0-C10.9). The study population included participants from Europe (45.3\%), North America (43.9\%) and South America (10.8\%). Details of the studies included, as well as the genotyping and imputation performed are published $^{30,37}$

Univariable Mendelian randomisation. To assess causal effects of smoking and alcohol consumption, we identified 60 single nucleotide polymorphisms (SNPs) for alcohol consumption and 176 SNPs for smoking initiation reaching genome-wide significance $\left(p<5 \times 10^{-8}\right)$ in the GSCAN GWAS which were present in the GAME-ON GWAS (Supplementary Data 1, Supplementary Data 2). We also identified 108 independent SNPs associated with the CSI at $p<5 \times 10^{-8}$ in the UK Biobank which were present in the GAME-ON GWAS (Supplementary Data 1, Supplementary Data 2).

Instrument strength was determined by the magnitude and precision of association of the genetic instruments with the risk factor, which was considered to be sufficient if the corresponding F-statistic is $>10$. Linkage disequilibrium statistics $\left(r^{2}\right)$ were evaluated to check the overlap between smoking initiation, comprehensive smoking index and drinks per week loci using LDmatrix. All pairwise correlations were low for the SNPs used to instrument smoking initiation and drinks per week $\left(r^{2}<0.06\right)$ and those used to instrument comprehensive smoking index and drinks per week $\left(r^{2}<0.07\right)$, with the exception of rs10236149 and rs6962772 which had an $r^{2}$ of 0.743 . For smoking initiation and comprehensive smoking index, there were 6 duplicate SNPs and 11 SNP pairs which were in strong $\operatorname{LD}\left(r^{2}>0.8\right)$.

Two-sample MR analyses were conducted using the TwoSampleMR package (version 0.5.5) in $\mathrm{R}$ (version 3.5.3), to extract the SNPs instrumenting the risk factor from the oral/oropharyngeal cancer $\mathrm{GWAS}^{38}$. We next performed harmonisation of the direction of effects between exposure and outcome 
associations, where palindromic SNPs were aligned when minor allele frequencies (MAFs) were less than 0.3 or were otherwise excluded. SNP-specific Wald estimates were calculated (SNP-outcome estimate divided by SNP-exposure estimate) and an inverse variance weighted (IVW) random effects method applied to meta-analyse these to obtain an estimate for the causal effect of the risk factor on oral/oropharyngeal cancer risk.

To further assess the robustness of findings, we inspected the Cochran's $Q$ statistic which assesses heterogeneity between individual genetic variants, and which may indicate the presence of invalid instruments (e.g., due to horizontal pleiotropy). Horizontal pleiotropy occurs when the genetic instruments are associated with more than one independent biological pathway, which can result in violation of the MR exclusion restriction assumption (i.e., the variable is not related to the outcome other than via the risk factor of interest). First-order inversevariance weights were used to calculate both the IVW estimate and Cochran's Q. A random effects model was used in the presence of heterogeneity ${ }^{39}$. Scatter and leave-one-out plots were produced to evaluate influential outliers and MR-PRESSO (Mendelian Randomisation Pleiotropy RESidual Sum and Outlier) was used to detect and correct for potential outliers $(p<0.05)^{40}$. MR-PRESSO is a unified framework that evaluates genetic pleiotropy in a standard MR model. It attempts to perform outlier removal in order to re-estimate the original exposure-outcome relationship to reduce bias in MR estimates. The IVW method will provide an unbiased estimate in the absence of horizontal pleiotropy or when horizontal pleiotropy is balanced ${ }^{41}$. To account for pleiotropy, we compared results with three other MR methods, which each make different assumptions about this: MREgger $^{42}$, weighted median ${ }^{43}$ and weighted mode ${ }^{44}$. MR-Egger can provide unbiased estimates even when all SNPs in an instrument violate the exclusion restriction assumption. Where there was evidence of violation of negligible measurement error (NOME), assessed based on the $I^{2}$ statistic, MR-Egger was performed with simulation extrapolation (SIMEX) correction ${ }^{45}$. SIMEX relies on simulation to estimate or reduce bias due to measurement error, considering additional data sets with increasing measurement error variance. The weighted median stipulates that at least $50 \%$ of the weight in the analysis stems from variants that are valid instruments ${ }^{43}$, while the weighted mode requires that the largest subset of instruments which identify the same causal effect to be valid instruments ${ }^{44}$

Stratification by cancer subsite. We further performed MR analysis for alcohol and smoking with stratification by cancer subsite to evaluate potential heterogeneity in the causal effects. For this we used GWAS summary data on a subset of 2641 oropharyngeal cases and 2990 oral cavity cases from the 6034 HNSCC cases in the GAME-ON Network GWAS used in the main analysis and the 6585 common controls ${ }^{30}$. A total of 954 individuals with HNSCC were excluded from this analysis since these were cases of hypopharynx and overlapping cancers.

We also conducted further sensitivity analyses to estimate causal effects using summary-level data obtained from the GWAS of smoking initiation and drinks per week in GSCAN excluding UK Biobank and 23andMe, and summary-level data obtained from a GWAS of smoking initiation ${ }^{38}$ and drinks per week ${ }^{13}$ in the UK Biobank only.

Multivariable Mendelian randomisation. We next conducted two-sample multivariable MR analysis which included SNPs which were genome-wide significant in either the GSCAN GWAS of alcohol consumption or the UK Biobank GWAS of comprehensive smoking index. After excluding SNPs with a pairwise $r^{2}$ greater than $0.001,168$ independent SNPs were used in the analysis. To remove sample overlap between the GWAS for alcohol consumption and CSI in the analysis, we used summary-level data obtained from the GWAS of drinks per week in GSCAN, excluding UK Biobank and 23andMe $(n=226,223)$ and summary-level data obtained from the GWAS of CSI in the UK Biobank $(n=462,690)$. Both the IVW and MR-Egger framework have been extended to estimate causal effects in multivariable MR analysis ${ }^{46,47}$, which was conducted using both the MVMR (version 0.2.0) and Mendelian Randomization ${ }^{48}$ (version 0.5.0) packages in R. We used generalised versions of Cochran's Q statistical tests to assess both instrument strength and validity in the two-sample summary data setting, where the covariance between the effects of the genetic variants on each exposure was fixed at zero by using non-overlapping samples for each exposure. This was performed using the MVMR package (version 0.2.0) in $\mathrm{R}$.

Investigating other risky behaviours. We used 124 SNPs associated with risk tolerance and 118 SNPs associated with lifetime number of sexual partners from a large GWAS meta-analysis ${ }^{13}$. We performed MR using the same univariable methods described in the main analysis and also evaluated the effects of these exposures stratified by cancer subsite.

Statistics and reproducibility. All genome-wide association study (GWAS) data used in this study had been previously replicated in independent datasets (see respective GWAS studies) $)^{9,12,30}$. In our study, MG and RR independently repeated all univariable and multivariable Mendelian randomisation analyses once, both successfully obtaining the same conclusions.
Reporting summary. Further information on research design is available in the Nature Research Reporting Summary linked to this article.

\section{Data availability}

GWAS summary statistics for the SNPs used to estimate the causal effects of smoking initiation, lifetime smoking and alcoholic drinks per week in this study are presented in Supplementary Data 1 and 2. Full summary statistics for the GAME-ON GWAS have been deposited in dbGAP (OncoArray: Oral and Pharynx Cancer; study accession number: phs001202.v1.p1 $)^{30}$. Published data from this study can also be found in: Lesseur, C. et al. Genome-wide association analyses identify new susceptibility loci for oral cavity and pharyngeal cancer. Nat Genet. 48, 1544-1550 (2016) ${ }^{30}$. Smoking initiation and alcohol consumption data (GSCAN study) are published in: Liu, M. Z. et al. Association studies of up to 1.2 million individuals yield new insights into the genetic aetiology of tobacco and alcohol use. Nat Genet. 51, $237(2019)^{9}$. Comprehensive smoking index (CSI) data is published in: Wootton, R. E. et al. Evidence for causal effects of lifetime smoking on risk for depression and schizophrenia: a Mendelian randomisation study. Psychol Med, 1-9 (2019) ${ }^{12}$. UK Biobank approval was given for this project (ID 40644-Investigating aetiology, associations and causality in diseases of the head and neck). A copy of the data generated in this this analysis is available at: https:// github.com/rcrichmond/smoking_alcohol_headandneckcancer ${ }^{49}$

\section{Code availability}

Two-sample MR analyses were conducted using the TwoSampleMR package (version 0.5.5) in $\mathrm{R}$ (version 3.5.3). Multivariable MR analysis was conducted using both the MVMR (version 0.2.0) and MendelianRandomization (version 0.5 .0$)^{48}$ packages in R. A copy of the code used in this this analysis is available at: https://github.com/rcrichmond/ smoking_alcohol_headandneckcancer ${ }^{49}$

Received: 18 February 2020; Accepted: 29 October 2020; Published online: 27 November 2020

\section{References}

1. Warnakulasuriya, S. Global epidemiology of oral and oropharyngeal cancer. Oral. Oncol. 45, 309-316 (2009).

2. Cancer Research UK. Head and Neck Cancer Statistics. https://www. cancerresearchuk.org/health-professional/cancer-statistics/statistics-bycancer-type/head-and-neck-cancers\#heading-Two (2019).

3. Ang, K. K. et al. Human papillomavirus and survival of patients with oropharyngeal cancer. N. Engl. J. Med. 363, 24-35 (2010).

4. Heck, J. E. et al. Sexual behaviours and the risk of head and neck cancers: a pooled analysis in the International Head and Neck Cancer Epidemiology (INHANCE) consortium. Int. J. Epidemiol. 39, 166-181 (2010).

5. Thomas, S. J., Penfold, C. M., Waylen, A. \& Ness, A. R. The changing aetiology of head and neck squamous cell cancer: A tale of three cancers? Clin. Otolaryngol. 43, 999-1003 (2018).

6. Hashibe, M. et al. Interaction between tobacco and alcohol use and the risk of head and neck cancer: pooled analysis in the International Head and Neck Cancer Epidemiology Consortium. Cancer Epidem. Biomar. 18, 541-550 (2009).

7. Smith, G. D. \& Ebrahim, S. 'Mendelian randomization': can genetic epidemiology contribute to understanding environmental determinants of disease? Int. J. Epidemiol. 32, 1-22 (2003).

8. Davey Smith, G. \& Hemani, G. Mendelian randomization: genetic anchors for causal inference in epidemiological studies. Hum. Mol. Genet. 23, R89-R98 (2014).

9. Liu, M. Z. et al. Association studies of up to 1.2 million individuals yield new insights into the genetic etiology of tobacco and alcohol use. Nat. Genet. 51, 237 (2019).

10. Burgess, S. \& Thompson, S. G. Multivariable Mendelian randomization: the use of pleiotropic genetic variants to estimate causal effects. Am. J. Epidemiol. 181, 251-260 (2015).

11. Sanderson, E., Davey Smith, G., Windmeijer, F. \& Bowden, J. An examination of multivariable Mendelian randomization in the single-sample and twosample summary data settings. Int. J. Epidemiol. 48, 713-727 (2018).

12. Wootton, R. E. et al. Evidence for causal effects of lifetime smoking on risk for depression and schizophrenia: a Mendelian randomisation study. Psychol. Med. 50 2435-2443 (2020).

13. Karlsson Linner, R. et al. Genome-wide association analyses of risk tolerance and risky behaviors in over 1 million individuals identify hundreds of loci and shared genetic influences. Nat. Genet. 51, 245-257 (2019).

14. Gillison, M. L., Chaturvedi, A. K., Anderson, W. F. \& Fakhry, C. Epidemiology of human papillomavirus-positive head and neck squamous cell carcinoma. $J$. Clin. Oncol. 33, 3235-3242 (2015). 
15. Vineis, P. et al. Tobacco and cancer: recent epidemiological evidence. J. Natl Cancer I 96, 99-106 (2004).

16. Bagnardi, V. et al. Alcohol consumption and site-specific cancer risk: a comprehensive dose-response meta-analysis. Br. J. Cancer 112, 580-593 (2015).

17. D'Souza, G. et al. Case-control study of human papillomavirus and oropharyngeal cancer. N. Engl. J. Med. 356, 1944-1956 (2007).

18. Gaudet, M. M. et al. Anthropometry and head and neck cancer: a pooled analysis of cohort data. Int. J. Epidemiol. 44, 673-681 (2015).

19. Chuang, S. C. et al. Diet and the risk of head and neck cancer: a pooled analysis in the INHANCE consortium. Cancer Res. 23, 69-88 (2009).

20. Khetan, P. et al. Occupations and the risk of head and neck cancer a pooled analysis of the International Head and Neck Cancer Epidemiology (INHANCE) Consortium. J. Occup. Environ. Med. 61, 397-404 (2019).

21. Rogers, M. A., Vaughan, T. L., Davis, S. \& Thomas, D. B. Consumption of nitrate, nitrite, and nitrosodimethylamine and the risk of upper aerodigestive tract cancer. Cancer Epidemiol. Biomark. Prev. 4, 29-36 (1995).

22. Wight, A. J. \& Ogden, G. R. Possible mechanisms by which alcohol may influence the development of oral cancer-a review. Oral. Oncol. 34, 441-447 (1998).

23. Olshan, A. F., Weissler, M. C., Watson, M. A. \& Bell, D. A. Risk of head and neck cancer and the alcohol dehydrogenase 3 genotype. Carcinogenesis 22, 57-61 (2001)

24. Dellarco, V. L. A mutagenicity assessment of acetaldehyde. Mutat. Res. 195, $1-20$ (1988).

25. Homann, N. et al. Effects of acetaldehyde on cell regeneration and differentiation of the upper gastrointestinal tract mucosa. J. Natl Cancer Inst. 89, 1692-1697 (1997).

26. Rothman, K. \& Keller, A. The effect of joint exposure to alcohol and tobacco on risk of cancer of the mouth and pharynx. J. Chronic Dis. 25, 711-716 (1972).

27. Boccia, S. et al. Aldehyde dehydrogenase 2 and head and neck cancer: a metaanalysis implementing a Mendelian randomization approach. Cancer Epidem. Biomar. 18, 248-254 (2009).

28. Ingold, N., Amin, H. A. \& Drenos, F. Alcohol causes an increased risk of head and neck but not breast cancer in individuals from the UK Biobank study: a Mendelian randomisation analysis. medRxiv 19002832 (2019).

29. Larsson, S. C. et al. Smoking, alcohol consumption, and cancer: a mendelian randomisation study in UK Biobank and international genetic consortia participants. PLoS Med. 17, e1003178 (2020).

30. Lesseur, C. et al. Genome-wide association analyses identify new susceptibility loci for oral cavity and pharyngeal cancer. Nat. Genet. 48, 1544-1550 (2016).

31. Salaspuro, V. \& Salaspuro, M. Synergistic effect of alcohol drinking and smoking on in vivo acetaldehyde concentration in saliva. Int. J. Cancer 111, 480-483 (2004).

32. Rees, J. M. B., Foley, C. N. \& Burgess, S. Factorial Mendelian randomization: using genetic variants to assess interactions. Int. J. Epidemiol. (2019).

33. Brennan, P. Commentary: Mendelian randomization and gene-environment interaction. Int. J. Epidemiol. 33, 17-21 (2004).

34. Millwood, I. Y. et al. Conventional and genetic evidence on alcohol and vascular disease aetiology: a prospective study of 500000 men and women in China. Lancet 393, 1831-1842 (2019).

35. Pierce, B. L. \& Burgess, S. Efficient design for Mendelian randomization studies: subsample and 2-sample instrumental variable estimators. Am. J. Epidemiol. 178, 1177-1184 (2013).

36. Lawlor, D. A. et al. A Mendelian Randomization dictionary: useful definitions and descriptions for undertaking, understanding and interpreting Mendelian randomization studies. OSF Preprints. https://doi.org/10.31219/osf.io/6yzs7 (2019).

37. Dudding, T. et al. Assessing the causal association between 25 -hydroxyvitamin $\mathrm{D}$ and the risk of oral and oropharyngeal cancer using Mendelian randomization. Int. J. Cancer 143, 1029-1036 (2018).

38. Hemani, G. et al. The MR-Base platform supports systematic causal inference across the human phenome. Elife 7, e34408 (2018).

39. Bowden, J. et al. Improving the visualization, interpretation and analysis of two-sample summary data Mendelian randomization via the Radial plot and Radial regression. Int. J. Epidemiol. 47, 1264-1278 (2018).

40. Verbanck, M., Chen, C. Y., Neale, B. \& Do, R. Publisher Correction: Detection of widespread horizontal pleiotropy in causal relationships inferred from Mendelian randomization between complex traits and diseases. Nat. Genet. 50, 1196 (2018).

41. Hemani, G., Bowden, J. \& Smith, G. D. Evaluating the potential role of pleiotropy in Mendelian randomization studies. Hum. Mol. Genet. 27, R195-R208 (2018).

42. Bowden, J., Davey Smith, G. \& Burgess, S. Mendelian randomization with invalid instruments: effect estimation and bias detection through Egger regression. Int. J. Epidemiol. 44, 512-525 (2015).
43. Bowden, J., Smith, G. D., Haycock, P. C. \& Burgess, S. Consistent estimation in mendelian randomization with some invalid instruments using a weighted median estimator. Genet. Epidemiol. 40, 304-314 (2016).

44. Hartwig, F. P., Smith, G. D. \& Bowden, J. Robust inference in summary data Mendelian randomization via the zero modal pleiotropy assumption. Int. J. Epidemiol. 46, 1985-1998 (2017).

45. Bowden, J. et al. Assessing the suitability of summary data for two-sample Mendelian randomization analyses using MR-Egger regression: the role of the I-2 statistic. Int. J. Epidemiol. 45, 1961-1974 (2016).

46. Rees, J. M. B., Wood, A. M. \& Burgess, S. Extending the MR-Egger method for multivariable Mendelian randomization to correct for both measured and unmeasured pleiotropy. Stat. Med. 36, 4705-4718 (2017).

47. Burgess, S., Dudbridge, F. \& Thompson, S. G. Re: "Multivariable Mendelian randomization: the use of pleiotropic genetic variants to estimate causal effects". Am. J. Epidemiol. 181, 290-291 (2015).

48. Yavorska, O. O. \& Burgess, S. MendelianRandomization: an R package for performing Mendelian randomization analyses using summarized data. Int. J. Epidemiol. 46, 1734-1739 (2017).

49. Gormley, M. et al. Multivariable Mendelian randomization to investigate the independent effects of smoking and alcohol on risk of head and neck cancer. GitHub. https://doi.org/10.5281/zenodo.4085102 (2020).

\section{Acknowledgements}

M.G. was a National Institute for Health Research (NIHR) academic clinical fellow and is currently supported by a Wellcome Trust GW4-Clinical Academic Training PhD Fellowship. This research was funded in part, by the Wellcome Trust [Grant number 220530/Z/20/Z]. For the purpose of Open Access, the author has applied a CC BY public copyright licence to any Author Accepted Manuscript version arising from this submission. M.G. also received a bursary from the Association of British Academic Oral and Maxillofacial Surgeons (ABAOMS) to help support this work. R.C.R. is a de Pass VC research fellow at the University of Bristol. J.T. is supported by an Academy of Medical Sciences (AMS) Springboard award, which is supported by the AMS, the Wellcome Trust, Global Challenges Research Fund (GCRF), the Government Department of Business, Energy and Industrial strategy, the British Heart Foundation and Diabetes UK (SBF004\1079). R.M.M. was supported by a Cancer Research UK (C18281/A19169) programme grant (the Integrative Cancer Epidemiology Programme) and is part of the Medical Research Council Integrative Epidemiology Unit at the University of Bristol supported by the Medical Research Council (MC_UU_12013/1, MC_UU_12013/2, and MC_UU_12013/3) and the University of Bristol. R.M.M. and A.R.N. are supported by the National Institute for Health Research (NIHR) Bristol Biomedical Research Centre which is funded by the National Institute for Health Research (NIHR) and is a partnership between University Hospitals Bristol and Weston NHS Foundation Trust and the University of Bristol. This publication presents data from the Head and Neck 5000 study. The study was a component of independent research funded by the NIHR under its Programme Grants for Applied Research scheme (RP-PG-0707-10034). The views expressed in this publication are those of the author(s) and not necessarily those of the NHS, the NIHR or the Department of Health. Core funding was also provided through awards from Above and Beyond, University Hospitals Bristol Research Capability Funding and the NIHR Senior Investigator award to A.R.N. B.D. and the University of Pittsburgh head and neck cancer case-control study are supported by US National Institutes of Health (NIH) grants: P50 CA097190, P30 CA047904 and R01 DE025712. The genotyping of the HNSCC cases and controls was performed at the Center for Inherited Disease Research (CIDR) and funded by the US National Institute of Dental and Craniofacial Research (NIDCR; 1X01HG007780-0). The University of North Carolina (UNC) CHANCE study was supported in part by the National Cancer Institute (R01-CA90731)

\section{Author contributions}

M.G., R.C.R. and T.D. conceived the study and all three carried out data curation and analysis, validating the results separately. Head and neck cancer summary genetic data was obtained through multiple collaborations from studies lead by A.R.N., S.T., A.F.O., R.J.H., G.L., B.D., S.B. and P.B. The initial manuscript was drafted by M.G., R.C.R. and T.D. Expert guidance on multivariable MR methodology was provided by E.S. and M.M provided specific advice on smoking and alcohol instruments such as the community smoking index analysis. G.D.S., J.T., S.T., M.M., P.B., R.M.M. and A.R.N. provided expertise on both observational and genetic epidemiological methodology and analysis. All authors M.G., T.D., E.S., R.M.M., S.T., J.T., A.R.N., P.B., M.M., M.P., S.B., A.F.O., R.J.H., G.L., B.D., G.D.S. and R.C.R. contributed to the interpretation of the results and critical revision of the manuscript. M.G. and this work was jointly supervised by R.C.R., J.T., A.R.N. and G.D.S

\section{Competing interests}

The authors declare no conflicts of interest. The funders had no role in the design of the study; the collection, analysis, and interpretation of the data; the writing of the manuscript; and the decision to submit the manuscript for publication. The authors alone are responsible for the views expressed in this article. 


\section{Additional information}

Supplementary information is available for this paper at https://doi.org/10.1038/s41467020-19822-6.

Correspondence and requests for materials should be addressed to M.G.

Peer review information Nature Communications thanks Verena Zuber, and the other, anonymous, reviewer for their contribution to the peer review of this work. Peer reviewer reports are available.

Reprints and permission information is available at http://www.nature.com/reprints

Publisher's note Springer Nature remains neutral with regard to jurisdictional claims in published maps and institutional affiliations. (c) (i) Open Access This article is licensed under a Creative Commons Attribution 4.0 International License, which permits use, sharing, adaptation, distribution and reproduction in any medium or format, as long as you give appropriate credit to the original author(s) and the source, provide a link to the Creative Commons license, and indicate if changes were made. The images or other third party material in this article are included in the article's Creative Commons license, unless indicated otherwise in a credit line to the material. If material is not included in the article's Creative Commons license and your intended use is not permitted by statutory regulation or exceeds the permitted use, you will need to obtain permission directly from the copyright holder. To view a copy of this license, visit http://creativecommons.org/ licenses/by/4.0/.

(C) The Author(s) 2020 